PROCEEDINGS OF THE

AMERICAN MATHEMATICAL SOCIETY

Volume 138, Number 11, November 2010, Pages 4059-4066

S 0002-9939(2010)10419-7

Article electronically published on May 18, 2010

\title{
AN IMPROVEMENT TO A BEREZIN-LI-YAU TYPE INEQUALITY
}

\author{
SELMA YILDIRIM YOLCU \\ (Communicated by Matthew J. Gursky) \\ This paper is dedicated to Professor Evans M. Harrell
}

\begin{abstract}
In this article we improve a lower bound for $\sum_{j=1}^{k} \beta_{j}$ (a BerezinLi-Yau type inequality) that appeared in an earlier paper of Harrell and Yolcu. Here $\beta_{j}$ denotes the $j$ th eigenvalue of the Klein Gordon Hamiltonian $H_{0, \Omega}=|p|$ when restricted to a bounded set $\Omega \subset \mathbb{R}^{n}$. $H_{0, \Omega}$ can also be described as the generator of the Cauchy stochastic process with a killing condition on $\partial \Omega$. To do this, we adapt the proof of Melas, who improved the estimate for the bound of $\sum_{j=1}^{k} \lambda_{j}$, where $\lambda_{j}$ denotes the $j$ th eigenvalue of the Dirichlet Laplacian on a bounded domain in $\mathbb{R}^{d}$.
\end{abstract}

\section{INTRODUCTION}

In this article, we consider the pseudodifferential operator $H_{0, \Omega}:=\sqrt{-\Delta}$ restricted to an open bounded set $\Omega$ in $\mathbb{R}^{d}$. This operator is sometimes called the fractional Laplacian with power $\frac{1}{2}$ (cf. [2] and [3]). We note that $H_{0, \Omega}$ is the generator of the Cauchy stochastic process with a killing condition on $\partial \Omega$ (cf. [2], [3]). Let $\beta_{k}$ denote the $k$ th eigenvalue of $H_{0, \Omega}$ and $u_{k}$ denote the corresponding normalized eigenfunction. Then the eigenvalues $\beta_{j}$ satisfy

$$
0<\beta_{1}<\beta_{2} \leq \beta_{3} \leq \cdots \leq \beta_{j} \leq \cdots \rightarrow \infty,
$$

where each eigenvalue is repeated according to its multiplicity. Throughout this article, $|\Omega|$ denotes the volume of the set $\Omega$. Consider the quadratic form

$$
Q(\varphi)=\int_{\Omega} \bar{\varphi} \sqrt{-\Delta} \varphi, \quad \varphi \in C_{c}^{\infty}(\Omega),
$$

where $\sqrt{-\Delta}$ is calculated for $\mathbb{R}^{d}$. Note that the quadratic form $Q$ is defined on a dense subset $C_{c}^{\infty}(\Omega)$ of $L^{2}(\Omega)$. By using the Fubini theorem and the definition of the Fourier transform and its inverse, it is easy to see that $Q$ is positive and symmetric. Since $\Omega \subset \mathbb{R}^{d}$ is non-empty, bounded and open, the operator $H_{0, \Omega}$ is defined as the Friedrichs extension ([1]) of the quadratic form $Q$ on $L^{2}(\Omega)$. Notice that $H_{0, \Omega}$ is the unique minimal positive operator extending $Q$.

Received by the editors September 19, 2009 and, in revised form, January 24, 2010.

2010 Mathematics Subject Classification. Primary 35P15; Secondary 35S99.

Key words and phrases. Fractional Laplacian, Weyl law, universal bounds, Klein-Gordon operator, Berezin-Li-Yau inequality. 
To show the analogy between the Dirichlet Laplacian and $H_{0, \Omega}$, we first mention similar results for the Dirichlet Laplacian. Let $\lambda_{j}$ be the eigenvalues of the Dirichlet Laplacian on $\Omega$. One such result is the so-called Li-Yau inequality proved by P. Li and S.-T. Yau. In [12, they proved that

$$
\sum_{j=1}^{k} \lambda_{j} \geq \frac{d C_{d}}{d+2}|\Omega|^{-2 / d} k^{1+2 / d},
$$

where $C_{d}=4 \pi \Gamma(1+d / 2)^{2 / d}$.

As mentioned in [11, (1.1) can be obtained by a Legendre transform of an earlier result by Berezin [5]. Hence, instead of calling it the Li-Yau inequality, we prefer the Berezin-Li-Yau inequality.

A.D. Melas improved the bound in the Berezin-Li-Yau inequality (1.1) in 14 and proved that

$$
\sum_{j=1}^{k} \lambda_{j} \geq \frac{d C_{d}}{d+2}|\Omega|^{-2 / d} k^{1+2 / d}+M_{d} k \frac{|\Omega|}{I(\Omega)}
$$

where the constant $M_{d}$ depends only on the dimension. Here $I(\Omega)$ is the moment of inertia, which is defined as $I(\Omega)=\min _{u \in \mathbb{R}^{d}} \int_{\Omega}|x-u|^{2} d x$.

An improvement to the last inequality (1.2) has recently been studied by many authors (cf. [10, [15], 9]). More precisely, in [10, H. Kovař́ík, S. Vugalter and T. Weidl improved (1.2) when $d=2$ and assuming geometric properties of the boundary of $\Omega$. Their proof is ingenious but somewhat intricate, and they first state and prove their result in the case of polygons, then in the case of general domains. Moreover, their result has a second term that has the order of $k$ as in the asymptotic behavior of the sum on the left hand side of (1.1):

$$
\sum_{j=1}^{k} \lambda_{j}=\frac{d C_{d}}{d+2}|\Omega|^{-2 / d} k^{1+2 / d}+\tilde{C}_{d} \frac{|\partial \Omega|}{|\Omega|^{1+1 / d}} k^{1+1 / d}+o\left(k^{1+1 / d}\right) \text { as } k \rightarrow \infty .
$$

As stated in [10], the correction term in (1.2) is of larger order than $k$, which appears in the asymptotics of (1.1).

The Riesz mean of order $\sigma$ is defined as

$$
R_{\sigma}(z)=\sum_{j}\left(z-\lambda_{j}\right)_{+}^{\sigma}
$$

Another analogous result is given in [15], where T. Weidl found a Berezin type bound for the Riesz mean $R_{\sigma}(z)$ when $\sigma>3 / 2$. The second term in this bound is similar to the second term in the asymptotics of $R_{\sigma}(z)$, up to a constant. His method utilizes sharp Lieb-Thirring inequalities for operator-valued potentials.

A natural question is how this approach can be adapted to the case of KleinGordon operators. This article answers this question and improves the Berezin-LiYau type bound in [8. We follow the basic strategy of [14, with some important differences of detail.

We first state the analogue of the Weyl asymptotic formula and the Berezin-LiYau type inequality in the case of Klein-Gordon operators $H_{0, \Omega}$. In 8 , E. Harrell and the author provided a different proof for the following asymptotic formula in [6]: 
Theorem 1.1 (Analogue of the Weyl asymptotic formula). As $k \rightarrow \infty$,

$$
\beta_{k} \sim \tilde{C}_{d}|\Omega|^{-1 / d} k^{1 / d},
$$

where $\tilde{C}_{d}=\sqrt{4 \pi} \Gamma(1+d / 2)^{1 / d}$.

This theorem can be proved by adapting a proof of the Weyl asymptotic formula for the Laplacian.

The analogue of the Berezin-Lie-Yau inequality shown in 8 reads:

Theorem 1.2 (Analogue of the Berezin-Lie-Yau inequality). The eigenvalues $\beta_{k}$ of $H_{0, \Omega}$ satisfy

$$
\sum_{j=1}^{k} \beta_{k} \geq \frac{d \tilde{C_{d}}}{d+1}|\Omega|^{-1 / d} k^{1+1 / d} .
$$

As in the original Li-Yau paper [12, the main tool used in the proof of this theorem is a generalization of the lemma which is attributed to Hörmander in [12. This result is also sharp in the sense of the Weyl asymptotic formula as in the case of the Laplacian.

\section{Statement AND PRoOF OF THE THEOREM}

The main result of this paper is given below.

Theorem 2.1. For $k \geq 1$ and the bounded set $\Omega$,

$$
\sum_{j=1}^{k} \beta_{j} \geq \frac{d \tilde{C}_{d}}{d+1}|\Omega|^{-1 / d} k^{1+1 / d}+\tilde{M}_{d} \frac{|\Omega|^{1+1 / d}}{I(\Omega)} k^{1-1 / d},
$$

where $\tilde{C}_{d}=\sqrt{4 \pi} \Gamma(1+d / 2)^{1 / d}$ and the constant $\tilde{M}_{d}$ depends only on the dimension $d$.

Observe that, in (1.2), the power of $k$ in the first term is $1+2 / d$ while in (2.1) the corresponding power is $1+1 / d$. This is not surprising because the KleinGordon operator can be viewed as the square root of the Laplacian in $\mathbb{R}^{d}$. Also, the improvement in (1.2) consists of $|\Omega| / I(\Omega)$ and in (2.1) we have $|\Omega|^{1+1 / d} / I(\Omega)$. Moreover, the difference between the powers of the $k$ terms on the right hand side of (2.1) is $2 / d$ as in (1.2).

It would be useful to compare the result with an expected two-term asymptotic expansion for the sum of the eigenvalues of $H_{0, \Omega}$ such as the formula (1.3). To the knowledge of the author, this has not been done before in the literature. However, R. Bañuelos, T. Kulczyski and B. Siudeja explored a similar result for the partition function $Z_{\Omega}(t)=\sum_{j=1}^{\infty} e^{-\beta_{j} t}$. See [4] for more details.

First, we will state and prove the following lemma, which is the crucial step in proving the theorem.

Lemma 2.2. Let $d \geq 2$ and $\varphi:[0, \infty) \rightarrow[0, \infty)$ be a decreasing, absolutely continuous function. Assume that

$$
0 \leq-\varphi^{\prime}(x) \leq m, \quad x>0 .
$$


Then,

$$
\begin{aligned}
\int_{0}^{\infty} x^{d} \varphi(x) d x & \geq \frac{1}{d+1}\left(d \int_{0}^{\infty} x^{d-1} \varphi(x) d x\right)^{1+1 / d} \varphi(0)^{-1 / d} \\
& +\frac{\varphi(0)^{2+1 / d}}{6 m^{2}\left(d^{2}-1\right)}\left(d \int_{0}^{\infty} x^{d-1} \varphi(x) d x\right)^{1-1 / d}
\end{aligned}
$$

Proof. Let us first define

$$
\eta(x)=\frac{1}{\varphi(0)} \varphi\left(\frac{\varphi(0)}{m} x\right) .
$$

Then $\eta(0)=1$ and $0 \leq-\eta^{\prime}(x) \leq 1$. To ease the notation, define $f(x):=-\eta^{\prime}(x)$ for $x \geq 0$. Hence, $0 \leq f(x) \leq 1$ for $x>0$ and $\int_{0}^{\infty} f(x) d x=\eta(0)=1$. Now, define

$$
A:=\int_{0}^{\infty} x^{d-1} \eta(x) d x \quad \text { and } \quad B:=\int_{0}^{\infty} x^{d} \eta(x) d x .
$$

Assume that $B<+\infty$, as otherwise the result is immediate. Thus, we can find a sequence $\left\{R_{j}\right\}$ such that $R_{j} \rightarrow \infty$ and $R_{j}^{d+1} \eta\left(R_{j}\right) \rightarrow 0$ as $j \rightarrow \infty$. Then, using integration by parts we get

$$
\int_{0}^{\infty} x^{d} f(x) d x=A d \quad \text { and } \quad \int_{0}^{\infty} x^{d+1} f(x) d x \leq(d+1) B .
$$

By the intermediate value theorem, there exist an $\alpha \geq 0$ such that

$$
\int_{\alpha}^{\alpha+1} x^{d-1} d x=(A d)^{1-1 / d}
$$

and

$$
\int_{\alpha}^{\alpha+1} x^{d+1} d x \leq \int_{0}^{\infty} x^{d+1} f(x) d x \leq(d+1) B .
$$

As we shall see later, the key point in the proof of the lemma is the inequality

$$
(d-1) x^{d+1}-(d+1) y^{2} x^{d-1}+2 y^{d+1} \geq 2 y^{d-1}(x-y)^{2}
$$

for $y>0$ and $x \geq 0$. The proof of (2.8) is straightforward. Indeed, first divide both sides by $y^{d+1}$. Then, by setting $\tau=x / y$ we get the polynomial

$$
\begin{aligned}
g(\tau) & :=(d-1) \tau^{d+1}-(d+1) \tau^{d-1}-2 \tau^{2}+4 \tau \\
& =\left((\tau-1)^{2} \tau\right)\left(\sum_{k=0}^{d-3}(2 k+4) \tau^{k}+(d-1) \tau^{d-2}\right) .
\end{aligned}
$$

An induction on $d$ gives the second equality, which leads to $g(\tau) \geq 0$. Now, integrating (2.8) from $\alpha$ to $\alpha+1$ and using (2.6) and (2.7) we get

$$
\begin{aligned}
(d+1)(d-1) B-(d+1) y^{2}(A d)^{1-1 / d}+2 y^{d+1} & \geq 2 y^{d-1} \int_{\alpha}^{\alpha+1}(x-y)^{2} d x \\
& \geq 2 y^{d-1} \int_{-1 / 2}^{1 / 2} s^{2} d s \\
& =\frac{y^{d-1}}{6} .
\end{aligned}
$$


Choosing $y=(A d)^{1 / d}$ yields

$$
B \geq \frac{1}{d+1}(A d)^{1+1 / d}+\frac{1}{6\left(d^{2}-1\right)}(A d)^{1-1 / d},
$$

or, equivalently,

$$
\begin{aligned}
\int_{0}^{\infty} x^{d} \eta(x) d x \geq & \frac{1}{d+1}\left(d \int_{0}^{\infty} x^{d-1} \eta(x) d x\right)^{1+1 / d} \\
& +\frac{1}{6\left(d^{2}-1\right)}\left(d \int_{0}^{\infty} x^{d-1} \eta(x) d x\right)^{1-1 / d}
\end{aligned}
$$

which together with (2.4) gives

$$
\begin{aligned}
\int_{0}^{\infty} x^{d} \varphi(x) d x \geq & \frac{1}{d+1}\left(d \int_{0}^{\infty} x^{d-1} \varphi(x) d x\right)^{1+1 / d} \varphi(0)^{-1 / d} \\
& +\frac{\varphi(0)^{2+1 / d}}{6 m^{2}\left(d^{2}-1\right)}\left(d \int_{0}^{\infty} x^{d-1} \varphi(x) d x\right)^{1-1 / d},
\end{aligned}
$$

concluding the proof.

Let us now prove Theorem 2.1 by using the lemma above.

Proof. Let the Fourier transform of each eigenfunction $u_{j}$ corresponding to the $j$ th eigenvalue $\beta_{j}$ be denoted by

$$
\hat{u}_{j}(\xi)=\frac{1}{(2 \pi)^{d / 2}} \int_{\Omega} e^{-i x \cdot \xi} u_{j}(x) d x .
$$

Since the set of eigenfunctions $\left\{u_{j}\right\}_{j=1}^{\infty}$ is an orthonormal set, the set of $\left\{\hat{u}_{j}(\xi)\right\}_{j=1}^{\infty}$ also forms an orthonormal set in $\mathbb{R}^{d}$ by using Plancherel's theorem. Set

$$
F(\xi):=\sum_{j=1}^{k}\left|\hat{u}_{j}(\xi)\right|^{2} .
$$

Now we will use the decreasing radial rearrangement of $F(\xi)$ and the coarea formula to get the condition in the lemma. Let $F^{*}(\xi)=\varphi(|\xi|)$ be the decreasing radial rearrangement of $F$. We may assume that $\varphi$ is absolutely continuous. Let $\mu(t)=$ $\left|\left\{F^{*}(\xi)>t\right\}\right|=|\{F(\xi)>t\}|$. Then, $\mu(\varphi(x))=\omega_{d} x^{d}$, where $\omega_{d}$ denotes the volume of the $d$-dimensional unit ball. By the coarea formula,

$$
\mu(t)=\int_{t}^{|\Omega| /(2 \pi)^{d}} \int_{\{F=x\}}|\nabla F|^{-1} d \sigma_{x} d x .
$$

Then,

$$
-\mu^{\prime}(\varphi(x))=\int_{\{F=\varphi(x)\}}|\nabla F|^{-1} d \sigma_{\varphi(x)} .
$$

Next we will estimate $|\nabla F|$ :

$$
\sum_{j=1}^{k}\left|\nabla \hat{u}_{j}(\xi)\right|^{2} \leq \frac{1}{(2 \pi)^{d}} \int_{\Omega}\left|i x e^{-i x \cdot \xi}\right|^{2} d x=\frac{I(\Omega)}{(2 \pi)^{d}},
$$


where $I(\Omega)$, the moment of inertia, is defined as

$$
I(\Omega)=\min _{u \in \mathbb{R}^{d}} \int_{\Omega}|x-u|^{2} d x .
$$

After translation, we may assume that

$$
I(\Omega)=\int_{\Omega}|x|^{2} d x .
$$

Observe that for every $\xi$,

$$
|\nabla F(\xi)| \leq 2\left(\sum_{j=1}^{k}\left|\hat{u}_{j}(\xi)\right|^{2}\right)^{1 / 2}\left(\sum_{j=1}^{k}\left|\nabla \hat{u}_{j}(\xi)\right|^{2}\right)^{1 / 2} \leq 2(2 \pi)^{-d} \sqrt{|\Omega| I(\Omega)}
$$

By letting $m:=2(2 \pi)^{-d} \sqrt{|\Omega| I(\Omega)}$ and using (2.11) in (2.10), we obtain

$$
\begin{aligned}
-\mu^{\prime}(\varphi(x)) & \geq m^{-1} \operatorname{Vol}_{n-1}(\{F=\varphi(x)\}) \\
& \geq m^{-1} d \omega_{d} x^{d-1} .
\end{aligned}
$$

On the other hand, differentiating $\mu(\varphi(x))$ yields $\mu^{\prime}(\varphi(x)) \varphi^{\prime}(x)=d \omega_{d} x^{d-1}$. Thus,

$$
0 \leq-\varphi^{\prime}(x) \leq m,
$$

which is the required condition in the lemma. Thus, it remains to prove the theorem by using the lemma. Observe that

$$
\int_{\mathbb{R}^{d}} F(\xi) d \xi=k
$$

Observe that because the $u_{j}$ 's form an orthonormal set in $L^{2}(\Omega)$, by Bessel's inequality,

$$
0 \leq F(\xi) \leq \frac{|\Omega|}{(2 \pi)^{d}}
$$

Since

$$
\beta_{j}=\left\langle u_{j}, H_{0, \Omega} u_{j}\right\rangle=\int_{\mathbb{R}^{d}}|\xi|\left|\hat{u}_{j}(\xi)\right|^{2} d \xi,
$$

with the definition of $F$, we have

$$
\int_{\mathbb{R}^{d}}|\xi| F(\xi) d \xi=\sum_{j=1}^{k} \beta_{j} .
$$

Hence,

$$
k=\int_{\mathbb{R}^{d}} F(\xi) d \xi=\int_{\mathbb{R}^{d}} F^{*}(\xi) d \xi=d \omega_{d} \int_{0}^{\infty} x^{d-1} \varphi(x) d x
$$

and

$$
\sum_{j=1}^{k} \beta_{j}=\int_{\mathbb{R}^{d}}|\xi| F(\xi) d \xi=\int_{\mathbb{R}^{d}}|\xi| F^{*}(\xi) d \xi=d \omega_{d} \int_{0}^{\infty} x^{d} \varphi(x) d x .
$$

Equations (2.16), (2.17), when combined with Lemma 2.2, yield

$$
\sum_{j=1}^{k} \beta_{j} \geq \frac{d}{d+1} \omega_{d}^{-1 / d} \varphi(0)^{-1 / d} k^{1+1 / d}+\frac{d}{6 m^{2}\left(d^{2}-1\right)} \omega_{d}^{1 / d} \varphi(0)^{2+1 / d} k^{1-1 / d}
$$


Define

$$
h(t)=\frac{d}{d+1} \omega_{d}^{-1 / d} k^{1+1 / d} t^{-1 / d}+\frac{C d}{m^{2}\left(d^{2}-1\right)} \omega_{d}^{1 / d} k^{1-1 / d} t^{2+1 / d},
$$

where $C$ is a constant to be chosen later. Observe that the function $h$ is decreasing on the interval

$$
0<t \leq\left(\frac{m^{2}(d-1) k^{2 / d}}{C(2 d+1) \omega_{d}^{2 / d}}\right)^{d /(d+2)} .
$$

Let $R$ be the number such that $|\Omega|=\omega_{d} R^{d}$. Then,

$$
I(\Omega) \geq \int_{B(R)}|x|^{2} d x=\frac{d \omega_{d} R^{d+2}}{d+2}
$$

where $B(R)$ is the ball of radius $R$. Then,

$$
\begin{aligned}
m & =2(2 \pi)^{-d} \sqrt{|\Omega| I(\Omega)} \\
& \geq 2(2 \pi)^{-d} \sqrt{\frac{d}{d+2} \omega_{d}^{-2 / d}|\Omega|^{(2 d+2) / d}} \\
& \geq(2 \pi)^{-d} \omega_{d}^{-1 / d}|\Omega|^{(d+1) / d} .
\end{aligned}
$$

Choosing $C=\min \left\{\frac{1}{6}, \frac{m^{2}(d-1) k^{2 / d}(2 \pi)^{d+2}}{(2 d+1) \omega_{d}^{2 / d}|\Omega|^{1+2 / d}}\right\}$ will guarantee that

$$
\left(\frac{m^{2}(d-1) k^{2 / d}}{C(2 d+1) \omega_{d}^{2 / d}}\right)^{d /(d+2)} \geq(2 \pi)^{-d}|\Omega|
$$

Hence, the function $h$ is decreasing on $\left(0,(2 \pi)^{-d}|\Omega|\right]$. Since $0<\varphi(0) \leq(2 \pi)^{-d}|\Omega|$, and $h$ is decreasing, we can replace $\varphi(0)$ in (2.18) with $(2 \pi)^{-d}|\Omega|$. Therefore, (2.18) and the fact that $\omega_{d}=\frac{\pi^{d / 2}}{\Gamma(1+d / 2)}$ result in the following inequality:

$$
\sum_{j=1}^{k} \beta_{j} \geq \frac{\sqrt{4 \pi} d k^{1+1 / d}}{d+1}\left(\frac{\Gamma(1+d / 2)}{|\Omega|}\right)^{1 / d}+\frac{C d k^{1-1 / d}}{8 \sqrt{\pi}\left(d^{2}-1\right)(\Gamma(1+d / 2))^{1 / d}} \frac{|\Omega|^{1+1 / d}}{I(\Omega)}
$$

Let $\tilde{M}_{d}:=\frac{C d}{8 \sqrt{\pi}\left(d^{2}-1\right)(\Gamma(1+d / 2))^{1 / d}}$. Then $(2.19)$ can be written as

$$
\sum_{j=1}^{k} \beta_{j} \geq \frac{d \tilde{C}_{d}}{d+1}|\Omega|^{-1 / d} k^{1+1 / d}+\tilde{M}_{d} \frac{|\Omega|^{1+1 / d}}{I(\Omega)} k^{1-1 / d}
$$

where $\tilde{C}_{d}=\sqrt{4 \pi} \Gamma(1+d / 2)^{1 / d}$. Recall that the first term on the right of (2.20) is the same bound as in 8 .

\section{ACKNOWLEDGEMENTS}

The author wishes to thank Evans Harrell, Lotfi Hermi, Rupert Frank, Türkay Yolcu and the referee of the paper for suggestions, comments and helpful discussions. 


\section{REFERENCES}

1. N.I. Akhiezer and I.M. Glazman, Theory of Linear Operators in Hilbert Space, Vols. I, II, Pitman, 1981. MR0615736 (83i:47001a) MR0615737 (83i:47001b)

2. R. Bañuelos and T. Kulczycki, The Cauchy process and the Steklov problem, J. Funct. Analysis 211(2) (2004) 355-423. MR2056835 (2005b:60124)

3. R. Bañuelos and T. Kulczycki, Eigenvalue gaps for the Cauchy process and a Poincaré inequality, J. Funct. Analysis 234(1) (2006) 199-225. MR.2214145 (2007c:60050)

4. R. Bañuelos, T. Kulczycki and Bartłomiej Siudeja, On the trace of symmetric stable processes on Lipschitz domains, J. Funct. Analysis 257(10) (2009) 3329-3352. MR2568694

5. F. A. Berezin, Covariant and contravariant symbols of operators, Izv. Akad. Nauk SSSR Ser. Mat. 36 (1972) 1134-1167. MR0350504 (50:2996)

6. R. Blumenthal and R. Getoor, The asymptotic distribution of the eigenvalues for a class of Markov operators, Pacific J. Math. 9 (1959) 399-408. MR0107298 (21:6023)

7. E. M. Harrell II and L. Hermi, Differential inequalities for Riesz means and Weyl-type bounds for eigenvalues, J. Funct. Analysis 254 (2008) 3173-3191. MR2418623 (2009f:47067)

8. E. M. Harrell II and S. Yildirim Yolcu, Eigenvalue inequalities for Klein-Gordon operators, J. Funct. Analysis, 256(12) (2009) 3977-3995. MR2521917

9. Alexei A. Ilyin, Lower bounds for the spectrum of the Laplace and Stokes operators, Discrete and Continuous Dynamical Systems, Volume 28, Number 1, September 2010, pp. 131-146. arXiv:0909.2818v1.

10. H. Kovař́k, S. Vugalter and T. Weidl, Two dimensional Berezin-Li-Yau inequalities with a correction term, Comm. Math. Phys., 287(3) (2009), 959-981. MR2486669

11. A. Laptev and T. Weidl, Recent results on Lieb-Thirring inequalities, Journées "Équations aux Dérivées Partielles" (La Chapelle sur Erdre, 2000), Exp. No. XX, 14 pp., Univ. Nantes, Nantes, 2000. MR.1775696 (2001j:81064)

12. P. Li and S.-T. Yau, On the Schrödinger equation and the eigenvalue problem, Comm. Math. Phys. 88 (1983) 309-318. MR701919 (84k:58225)

13. E. H. Lieb and M. Loss, Analysis, second edition. Graduate Studies in Mathematics 14, Providence, RI: Amer. Math. Soc. (2001). MR1817225 (2001i:00001)

14. A. D. Melas, A lower bound for sums of eigenvalues of the Laplacian, Proceedings of the American Mathematical Society 131(2) (2003) 631-636. MR1933356 (2003i:35218)

15. T. Weidl, Improved Berezin-Li-Yau inequalities with a remainder term, in Spectral Theory of Differential Operators, Amer. Math. Soc. Transl. Ser. 2, 225, Providence, RI: Amer. Math. Soc. (2008), 253-263. MR2509788

School of Mathematics, Georgia Institute of Technology, Atlanta, Georgia 30332

Current address: Department of Mathematics, Georgia College \& State University, Milledgeville, Georgia 31061; (after August 2010) Department of Mathematics, Purdue University, West Lafayette, Indiana 47907

E-mail address: selma@math.gatech.edu, selma.yildirim-yolcu@gcsu.edu 